\title{
Efecto del pulido radicular en la adhesión dentinaria: estudio al microscopio electrónico de barrido (MEB)
}

\section{Effects of the root planning in the dentin adhesión: a SEM study}

\author{
VALENZUELA ARÁNGUIZ V* \\ BOFILL FONTBOTÉ $S$ ** \\ KOTTMANN LEDESIMA C*** \\ FODICH SATTA I*** \\ BRUNET ECHAVARRÍA J*
}

Valenzuela Aránguiz V, Bofill Fontboté S, Kottmann Ledesma C, Fodich Satta I, Brunet Echavarría J. Efecto del pulido radicular en la adhesión dentinaria: estudio al microscopio electrónico de barrido (MEB). Av Periodon Implantol. 2010; 22, 1: 19-25

\begin{abstract}
RESUMEN
Para analizar al MEB las características micromorfológicas de la adhesión dentinaria en superficies radiculares expuestas a enfermedad periodontal y tratadas con pulido radicular, se utilizaron 30 dientes, separados en tres grupos: Grupo 1: Dientes con enfermedad periodontal tratados solo con pulido radicular. Grupo 2: Dientes con enfermedad periodontal tratados con pulido radicular y sometidas a grabado con ácido fosfórico al 34\% por dos minutos. Grupo 3: Dientes con enfermedad periodontal tratados con pulido radicular, en las cuales se realizó todo el protocolo adhesivo para confeccionar resinas compuestas. Resultados. Grupo 1: Se observó una capa de barro dentinario en toda la superficie. Grupo 2: Presentaba una irregular capa de barro dentinario. Grupo 3: Se observó una seudocapa híbrida de $15,9 \mu \mathrm{m}$ de grosor, pero no se evidenció la presencia de tags de resina. Conclusiones. La adhesión en raíces con enfermedad periodontal y tratadas con pulido radicular se realiza sobre barro dentinario que no es removido por la acción del ácido fosfórico, no formándose capa híbrida ni tags de resina, por lo tanto creemos que la adhesión como concepto propiamente tal no existe en estos tejidos.
\end{abstract}

PALABRAS CLAVE: Adhesión, capa híbrida, pulido radicular, barro dentinario, dentina radicular.

\section{SUIMMARY}

The aim of this in vitro study was to analize some characteristics of dentinary adhesion over root surfaces exposed to periodontal disease an treated with root planning by observing the samples at SEM. 30 teeth were classified in three groups. Group 1: Teeth with periodontal disease treated only by root planning. Group 2: Teeth with periodontal disease treated by root planning and $34 \%$ fosforic acid etching. Group 3: Teeth with periodontal disease treated by root planning at and an adhesive composite restoration. Results: Group 1 presented a thick smear layer over all the treated surface. Group 2 presented an irregular adhesive layer. Group 3 showed a $15.9 \mu \mathrm{m}$ thick layer that looks as an hybrid layer, composite tags were not found in this group. Conclusions: Dentine adhesion over teeth with periodontal disease and treated by root planning is achieved over a thick smear layer, which is not removed by fosforic acid. Hybrid layer and composite tags were not formed, so it is assumed that adhesion does not occur.

KEY WORDS: Adhesion, hybrid layer, root planning, smear layer, root dentin.

Fecha de recepción: 14 de julio de 2008.

Fecha de aceptación: 21 de julio de 2008.

\footnotetext{
* $\quad$ Profesor Dpto. Odontología Integral del Adulto. Fac. Odontología. U. Mayor. Chile.

** Instructor Dpto. Odontología Integral del Adulto. Fac. Odontología. U. Mayor. Chile.

*** Cirujano-Dentista. Práctica privada.
} 


\section{INTRODUCCIÓN}

La Odontología moderna ha hecho posible el desarrollo de nuevas técnicas aplicadas a la rehabilitación, en donde la estética adquiere gran importancia en las demandas y expectativas de nuestros pacientes. Con el desarrollo de la adhesión se ha generado una revolución en la Odontología Restauradora y a pesar de las grandes ventajas que implica su aplicación clínica, no ha estado exenta de problemas. En la dentina se han observado las mayores dificultades en cuanto a adhesión, ya que este tejido posee un gran contenido orgánico, conformado principalmente por colágeno tipo I (1-3). Esta adhesión es básicamente micromecánica, basada en la hibridación de la dentina intertubular y en la formación de tags de resina (4), que dependen fundamentalmente de la permeabilidad dentinaria y la difusión de monómeros en la malla colágena expuesta, que aportan a los importantes valores de adhesión logrados en este sustrato (5). Uno de los factores que puede alterar la permeabilidad dentinaria es el barro dentinario (smear layer) (6), el cual está compuesto por tejido calcificado, material orgánico e inorgánico, que se forma luego que la dentina es cortada con instrumentos rotatorios o manuales (7).

El procedimiento de destartraje y pulido radicular que se realiza como parte del tratamiento de la Enfermedad Periodontal, es el método más eficiente para la remoción de placa bacteriana y cemento contaminado sin llegar a eliminarlo completamente. Clínicamente pueden quedar túbulos dentinarios prácticamente descubiertos al medio bucal, sin embargo, estudios in vitro han observado la presencia de túbulos dentinarios obliterados por barro dentinario posterior al tratamiento periodontal $(7,8)$.

A lo anteriormente expuesto se debe considerar que a nivel radicular, la dentina se caracteriza por ser menos permeable, ya que posee un menor número de túbulos dentinarios y de menor diámetro $(1,2,3,9)$, con lo cual la adhesión podría verse afectada por la reducción de la permeabilidad dentinaria a este nivel. El propósito de este trabajo es determinar las características micromorfológicas que se establecerían en la adhesión a la dentina radicular en dientes con Enfermedad Periodontal tratados con pulido radicular.

\section{MATERIAL Y MÉTODO}

Para la realización de esta investigación, se utilizaron 30 dientes con indicación de extracción por enferme- dad periodontal severa, almacenados en suero fisiológico al $9 \%$ a temperatura ambiente hasta el momento de su utilización, posteriormente fueron asignadas aleatoriamente en tres grupos:

- Grupo 1: Dientes con enfermedad periodontal tratados solo con pulido radicular.

- Grupo 2: Dientes con enfermedad periodontal tratados con pulido radicular y sometidos a grabado con ácido fosfórico al $34 \%$ por 2 minutos.

- Grupo 3: Dientes con enfermedad periodontal tratados con pulido radicular, en los cuales se prepararon resinas compuestas en la superficie radicular cervical siguiendo los protocolos adhesivos.

El primer procedimiento desarrollado fue el pulido radicular manual de todas las muestras, desde el límite amelocementario hasta $6 \mathrm{~mm}$ hacia apical, utilizando curetas periodontales específicas tipo Gracey $\mathrm{N}^{\circ}$ 7-8 y 5-6. Esta etapa fue realizada por un operador calibrado, quien consideraba terminada la instrumentación de las superficies radiculares, una vez que éstas se encontrasen suaves y sin depósitos duros.

Para el grupo 2, las superficies radiculares fueron instrumentadas biomecánicamente y posteriormente se grabó la superficie con ácido fosfórico al $34 \%$ por dos minutos y se lavó profusamente con agua por dos minutos.

En el grupos 3, los dientes se restauraron, sin realizar cavidades, con resinas compuestas (Filtex Supreme, 3M-Espe), desde el límite amelocementario hasta $6 \mathrm{~mm}$ hacia apical. Para ello se acondicionó la superficie, grabando con ácido fosfórico al $34 \%$ por 15 segundos, seguido de un lavado profuso con agua por 15 segundos y la eliminación del exceso de esta mediante un soplado suave con aire. Utilizando un micropincel, se aplicó sobre estas superficies una capa de adhesivo monocomponente Single Bond (3M-ESPE), dejándolo actuar por 10 segundos y eliminando los excesos con aire. Posteriormente se aplicó una segunda capa de adhesivo, repitiendo el mismo procedimiento mencionado, fotopolimerizándolas por $20 \mathrm{se}-$ gundos. Finalmente, se aplicó una capa de resina compuesta de nanorrelleno, (Filtex Supreme, 3M-Espe) a modo de restauración.

Para poder observar el resultado de los procedimientos realizados con el microscopio electrónico de barrido, en las muestras de los grupos 1 y 2 se realizaron cortes transversales de los dientes y se conformaron probetas de $4 \mathrm{~mm}$ de ancho por $5 \mathrm{~mm}$ de grosor. El procesamiento para el grupo 3 fue diferente, pues 2 
$\mathrm{mm}$ de grosor correspondían a la preparación de resina compuesta y $3 \mathrm{~mm}$ al tejido dentinario, de tal forma poder observar la interfase dentina-restauración, estas muestras se pulieron con lijas al agua y discos soflex extra fino, hasta obtener una superficie muy lisa (a espejo). Con el propósito de eliminar el barro dentinario generado por el corte de las fresas y el pulido con lijas y discos, se grabó con ácido fosfórico al 34\%, seguido por un profuso lavado con agua.

Todas las muestras se fijaron en solución de glutaraldehido $2,5 \%$ tamponado a $\mathrm{pH} \mathrm{7,4}$ con fosfato $0,1 \mathrm{M}$. por 24-48 horas, luego fueron sometidas a proceso de deshidratación con acetona. Posteriormente fueron secadas por punto crítico, montadas en soportes y metalizadas con Oro-Paladio para la observación al microscopio electrónico de barrido (Zeiss DSM 940).

\section{RESULTADOS}

En las siguientes microfotografías se observan los resultados mas representativos obtenidos con los grupos en estudio y a diferentes aumentos.

- Grupo 1. Superficies radiculares sometidas sólo a pulido radicular (Figs. 1 y 2).

- Grupo 2. Superficies radiculares sometidas a pulido radicular y grabado ácido por 2 minutos.

Las superficies radiculares de todas las muestras pertenecientes al grupo 2, se caracterizaron por la presencia y persistencia de una capa irregular de barro dentinario agrietado, a pesar del grabado ácido, tal como se muestra en las figuras 3 y 4 .

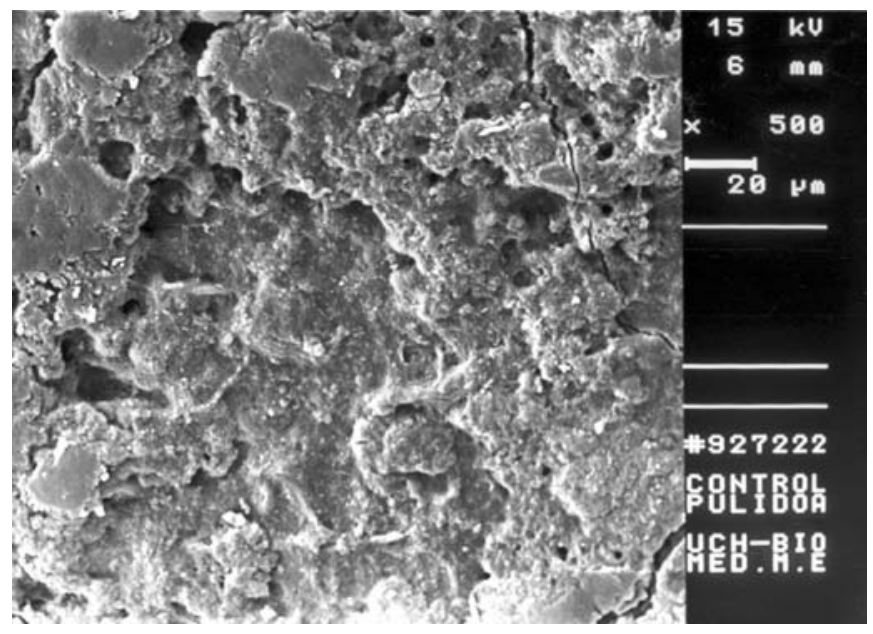

Fig. 1.

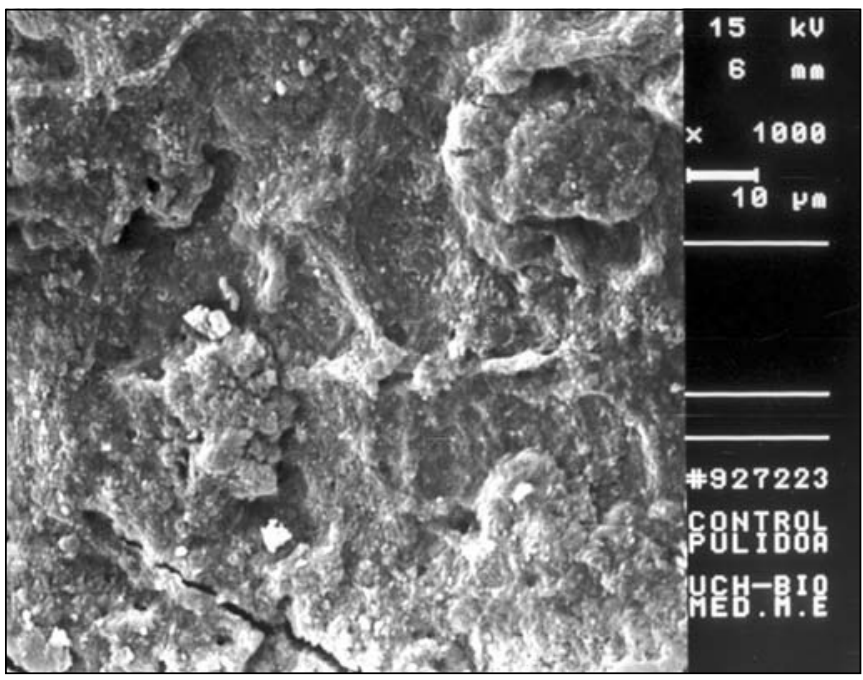

Figs. 1 y 2. A estos aumentos (500× y 1.000×) se observan las características irregulares del barro dentinario formado sobre las superficies radiculares después del pulido.

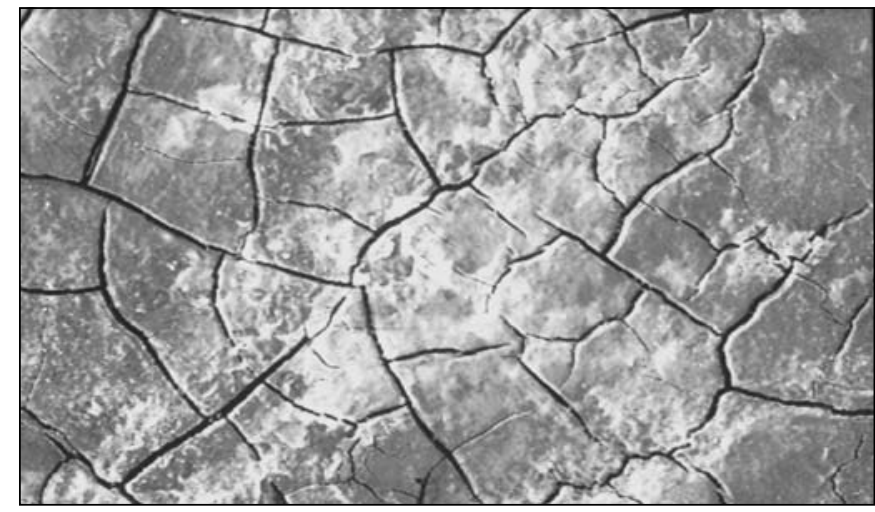

Fig. 3. Barro dentinario encontrado posterior a la instrumentación biomecánica y grabado con ácido fosfórico (50 ×).

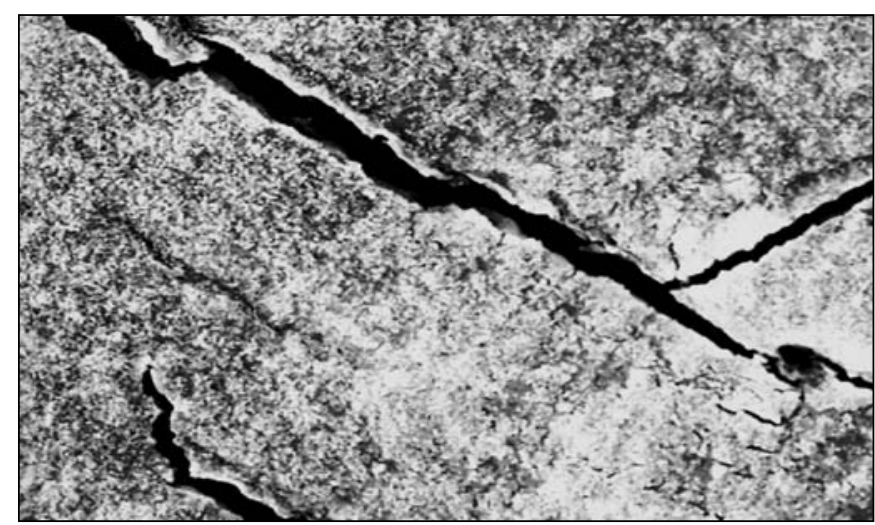

Fig. 4. Barro dentinario a mayor aumento (500x). 
- Grupo 3. Superficies radiculares expuestas a enfermedad periodontal con pulido radicular y protocolo adhesivo.

En este grupo se observó una densa y gruesa capa seudoadhesiva, que se formó en todas las muestras. No se encontró formación de tags de resina en ninguna de ellas (figs. 5-7).

\section{Grosor de la capa adhesiva del grupo 3}

El grosor presentado por esta seudocapa adhesiva de las muestras fue agrupado en la tabla l, dando un promedio de $15,9 \mu \mathrm{m}$.
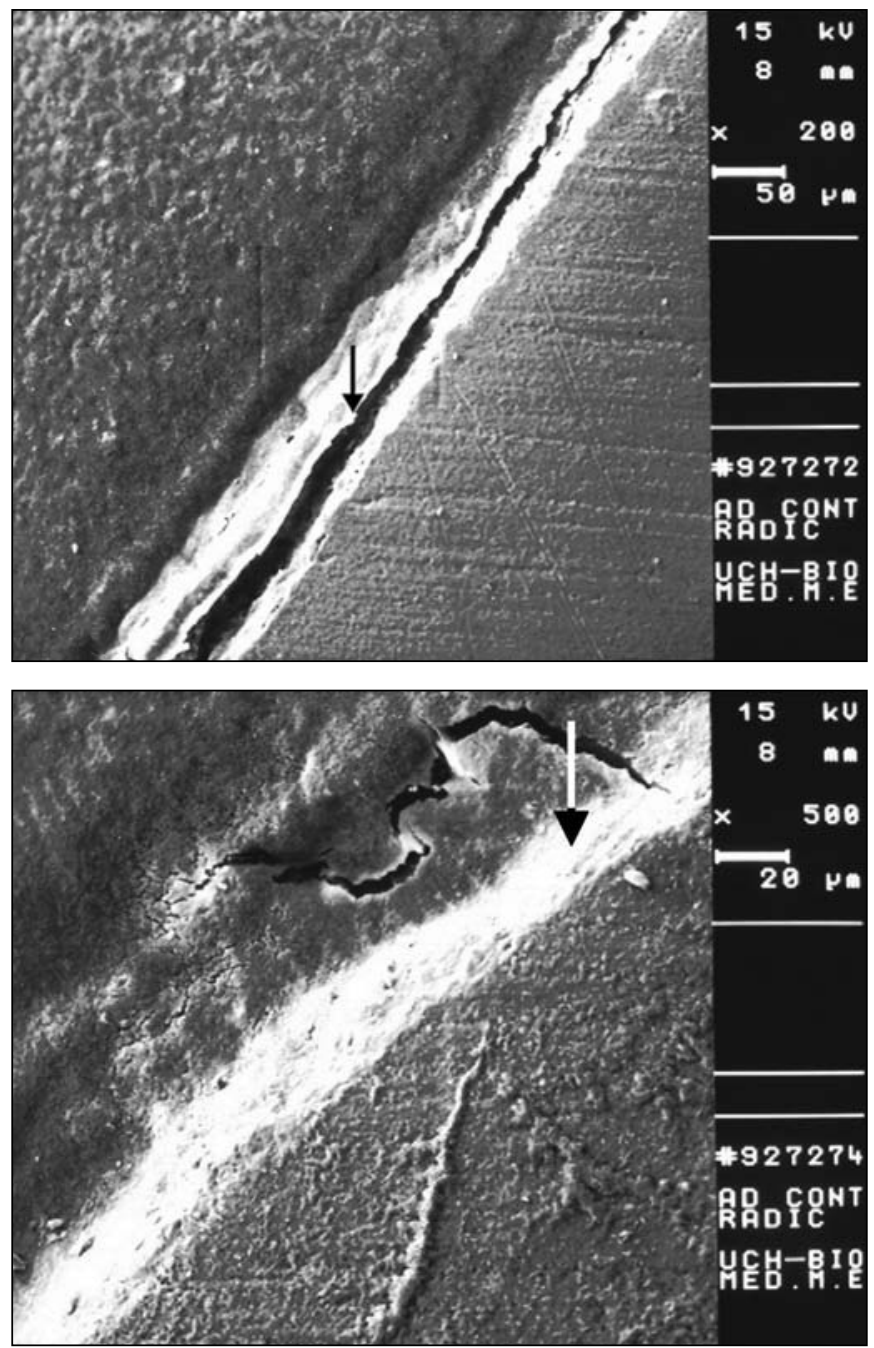

Figs. 5 y 6. En la figura 5, a 200×, se observa una separación entre la resina compuesta y el tejido dentinario radicular (flecha). En la figura 6, a 500×, se aprecia el grosor de esta seudocapa híbrida (flecha).

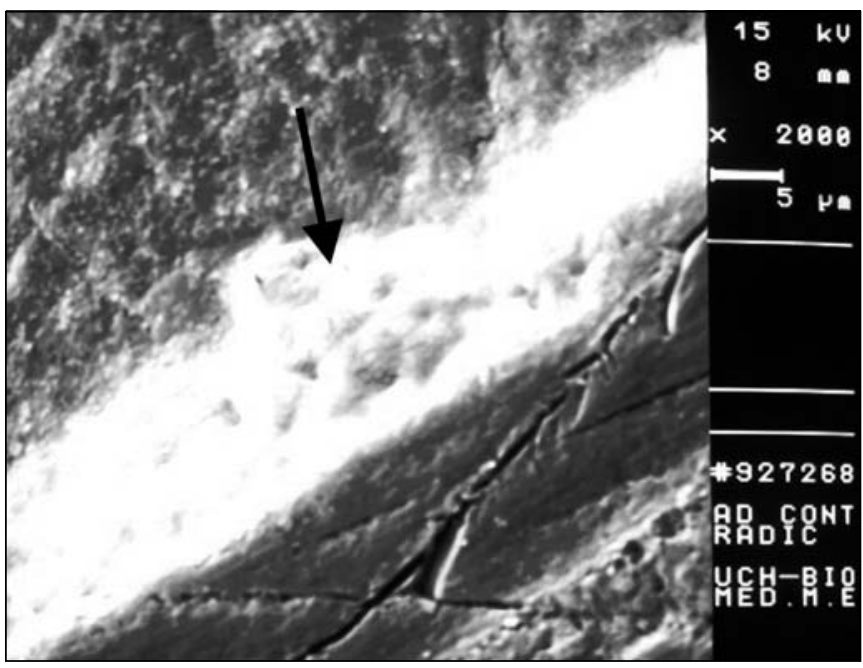

Fig. 7. A mayor aumento se observa el grosor que presenta en algunas zonas esta seudocapa adhesiva (2.000×).

\begin{tabular}{|cc|}
\hline \multicolumn{2}{|c|}{$\begin{array}{c}\text { TABLA } \\
\text { ADHESIVA DEL GRUPO 3 }\end{array}$} \\
\hline $\mathbf{N}^{\circ}$ de muestras & Grosor de capa adhesiva $(\boldsymbol{\mu m})$ \\
\hline 1 & 16,6 \\
\hline 2 & 12,5 \\
\hline 3 & 20 \\
\hline 4 & 25 \\
\hline 5 & 10 \\
\hline 6 & 15 \\
\hline 7 & 16,6 \\
\hline 8 & 12,1 \\
\hline 9 & 15,5 \\
\hline 10 & 16,1 \\
\hline Promedio & 15,9 \\
\hline
\end{tabular}

\section{DISCUSIÓN}

Al observar las muestras de dientes afectados por enfermedad periodontal y tratadas con pulido radicular se encontró que todas ellas se encontraban contaminadas con barro dentinario, generado por la instrumen- 
tación biomecánica, caracterizada por ser una capa gruesa, compacta e irregular, en el cual resultó imposible determinar sus componentes, y por ende no se pudo distinguir si posterior a la instrumentación quedaba algún resquicio de cemento. Solamente se puede afirmar que no existió ninguna zona en que aparentemente se observara algún túbulo dentinario expuesto.

Esta situación es coincidente con las observaciones planteadas por Blomlöf et al (1997), quienes afirmaron que resultaba imposible descontaminar completamente una superficie radicular afectada por Periodontitis utilizando únicamente medios mecánicos, ya que la superficie instrumentada inevitablemente se vería cubierta de este barro dentinario. Además agregaron, que dicho barro era lábil a la acción de los ácidos (8).

En la práctica odontológica, el ácido más ocupado para el acondicionamiento dentinario previo a la adhesión de resinas compuestas es el ácido fosfórico. A modo de saber si el barro dentinario generado posterior al pulido radicular era lábil a su acción, se utilizó en algunas muestras ácido fosfórico por 2 minutos, sin embargo, nuevamente se encontró una densa capa de barro, lo que indicaba que se estaba frente a un sustrato con propiedades de solubilidad distintas a las estudiadas por otros investigadores. Esta observación concuerda con las conclusiones de otras publicaciones en las que se encontró que el barro dentinario a nivel radicular no era fácil de grabar y tenía un gran componente orgánico en comparación con barros de otras superficies dentarias (11). Este último aspecto es muy importante para la infiltración de resinas en la superficie dentinaria acondicionada, ya que un sustrato orgánico sobre dicha superficie, implicaría una fuerte disminución en su energía superficial, lo que reduciría notablemente la fuerza adhesiva de las resinas con el tejido dentinario.

Fogel y Pashley (1993), no concuerdan con el concepto de un barro insoluble a la acción de ácidos, por lo contrario, determinaron que es lábil a la acción de éstos, y demostraron la susceptibilidad del barro a la acción de sustancias de la dieta, enjuagues bucales, entre otros agentes, por lo que no existirían problemas en su disolución (10).

Con respecto a esta problemática, existen una serie de factores implicados, que pueden influir en la solubilidad del barro dentinario, como por ejemplo si éste es ácido resistente, o si el tipo o concentración del ácido es el correcto a utilizar. Por ejemplo, se han utilizado una serie de agentes para la remoción de dicho barro, tales como sales, detergentes, ácido cítrico, tetraciclinas, etc., que además tienen el efecto de desmineralizar y exponer la malla de colágeno del tejido radicular; sin embargo la aplicación clínica de estos productos tienen un objetivo distinto, que es permitir la migración o unión de células, que son necesarias para la reparación espontánea y óptima del tejido periodontal de soporte, por medio de la generación de una superficie radicular biocompatible, libre de contaminantes.

Así también, otro factor importante a considerar para la eliminación de dicho barro, es el grosor que éste puede alcanzar, ya que el ácido es capaz de desmineralizar la superficie dentaria entre 0,5 a $5 \mu \mathrm{m}$., de superarse esta cifra (5), la posible solución sería utilizar el ácido por etapas, o sea grabar por un minuto, lavar y volver a grabar la superficie por segunda o tercera vez, de manera de ir disminuyendo el grosor del barro, el cual puede alcanzar un espesor 10 a $15 \mu \mathrm{m}$.

Otro aspecto a tener en cuenta es el tejido dentinario propiamente tal, a saber, una de las características de la dentina radicular, esencial para la adhesión de resinas compuestas, es la menor permeabilidad que ésta tiene en comparación con la dentina coronaria, ya que presenta un menor diámetro y número de túbulos dentinarios por $\mathrm{mm}^{2}$. Esta situación se acentúa aún más cuando se está en presencia de una superficie radicular expuesta a una serie de irritantes, como las endotoxinas bacterianas, provenientes de la placa bacteriana y cálculo dental, que obligan a generar una respuesta, traducida en un cambio composicional y microestructural del tejido dentinario, manifestado en una esclerosis, que se caracteriza por la aposición de dentina peritubular, áreas de hipermineralización, ausencia o disminución de túbulos dentinarios, situación que podría llegar a ser irreversible aún cuando estos sean grabados con ácidos, lo que implica una mayor reducción en la permeabilidad dentinaria. Este tipo de dentina contiene, en su estructura, cristales de whitlockita; altamente resistente al ataque ácido, por tanto es razonable predecir que los adhesivos dentinarios son menos exitosos cuando son aplicados en dentina esclerótica al compararla con dentina normal. Pashley y Carvallo (1997) señalaron que en una revisión de la literatura sobre adhesión dentinaria, la resistencia a la tracción en dentina esclerótica es un $30 \%$ más baja que en dentina sana (12).

En base a estos antecedentes, y a modo de corroborar - descartar las características presentes en estas superficies radiculares expuestas, se optó por observar 
dicho tejido un poco más allá de la superficie misma de las muestras, encontrándose dentina de tipo esclerótica donde la observación de los túbulos dentinarios era difícil. (ver figura 8).

En las muestras del Grupo 3 se encontró la formación de una seudocapa híbrida bastante más gruesa que lo encontrado normalmente a nivel coronario $(4-7 \mu \mathrm{m})$, cuyo promedio fue de $15,9 \mu \mathrm{m}$ de grosor. Pero si se considera que la superficie radicular tratada con pulido radicular se caracteriza por presentar una gruesa capa de barro dentinario, lo que realmente se observa no corresponden al concepto capa híbrida específicamente, y además no garantizan una adhesión estable, ya que primero, se trata de un sustrato con alto contenido orgánico, con baja energía superficial y su unión a la superficie dentaria no sería confiable, cabe destacar además, que no se encontró presencia de tags de resina en ninguna de las muestras observadas.

Como colofón podemos decir que la dentina radicular es más mineralizada que la dentina coronaria y posee un menor grado de permeabilidad, lo que hace que sea un sustrato más difícil para la adhesión, factores que se deben tener en cuenta al momento de realizar procedimientos de adhesión a nivel radicular. Más aún, las superficies radiculares expuestas a periodontitis y tratadas con pulido radicular parecen incrementar las dificultades como un sustrato adecuado para la adhesión de resinas compuestas, por ello el tratamiento de

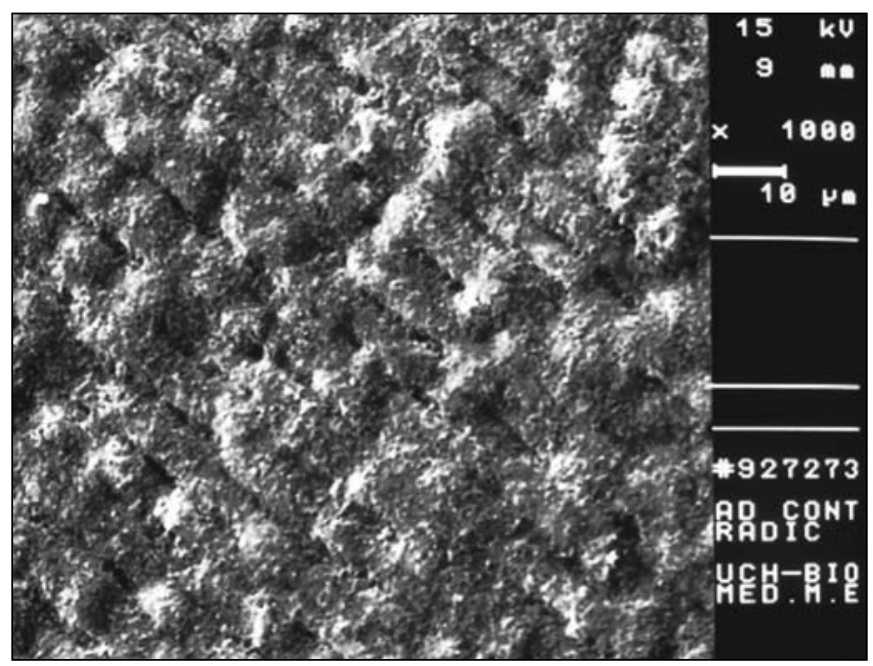

Fig. 8. Se aprecia, a 1.000x, un corte longitudinal de dentina radicular, de un diente afectado por enfermedad periodontal, presentándose altamente esclerosada. estas superficies ha de ser estudiado más profundamente.

\section{CONCLUSIONES}

1. Las superficies radiculares con enfermedad periodontal tratadas con pulido radicular se caracterizan por presentar una gruesa capa de barro dentinario.

2. El barro dentinario generado posterior a la instrumentación periodontal no es removido completamente por la acción del ácido fosfórico.

3. No se forman capa híbrida ni tags de resinas compuestas en las superficies radiculares con enfermedad periodontal y tratadas con pulido radicular, por lo tanto no se genera adhesión.

\section{BIBLIOGRAFÍA}

1. Abramovich. Histología y embriología dentaria. Segunda edición. Capítulo 3. Editorial Panamericana. Buenos Aires. 1999:42-73.

2. Gómez de Ferraris M, Campos Muñoz A: Histología y Embriología bucodental. Capitulo 9. Editorial Panamericana, 1999: 238-69.

3. Montenegro MA y cols.: Histología y embriología del Sistema estomatognático. Ediciones Facultad de Odontología Universidad de Chile. Santiago. 1997:70-81.

4. Tanumiharja M, Burrow MF, Tyas MJ, Carpenter J. Fieldemission scanning electron microscopy of resin-dentin interface morphology of seven dentin adhesive systems J Adhes Dent. 2000; 2 (4):259-69.

5. Van Meerbeek B, Inoue S, Perdigaô J, Lambrechts P. et al. Enamel and Dentin Adhesion. En: Fundamental of Operative Dentistry. Segunda edición. Chicago. Quintessence Publishing, 2001:178-222.

6. Mohan B, Kandaswamy D. A confocal microscopic evaluation of resin-dentin interface using adhesive systems with three different solvents bonded to dry and moist dentinan in vitro study Quintessence Int. 2005;36 (7-8):511-21.

7. Sampaio JE, Theodoro LH, Correa MA, Mendes AJ. A comparative SEM study of smear layer removal by 
detergents and EDTA on the root surface. Int J Periodontics Restorative Dent. 2005; 25(2):157-63.

8. Blomlof JP, Blomlof LB, Lindskog SF. Smear layer formed by different root planing modalities and its removal by an ethylenediaminetetraacetic acid gel preparation. Int J Periodontics Restorative Dent. 1997; 17(3):242-9.

9. Van Meerbeek B,De Munk,Yoshida, et al.Buonocore Memorial Lecture. Adhesion to Enamel and Dentin: Current status and Future challenges, Oper Dent. 2003;28(3):215-35.

10. Fogel HM, Pashley DH. Effect of periodontal root planing on dentin permeability. J Clin Periodontol. 1993 Oct;20 (9):673-7.
11. Kerns DG, Scheidt MJ, Pashley DH, Horner JA, Strong SL, Van Dyke TE. Dentinal tubule occlusion and root hypersensitivity. J Periodontol. 1991;62(7):421-8.

12. Pashley DH. and Carvalho RM. Dentine permeability and dentin adhesion. J. Dent. 1997; 25: 355-72.

\section{CORRESPONDENCIA}

Vladimir Valenzuela Aránguiz Las Bellotas 199 of. 75, Providencia, Santiago de Chile

e-mail: vladyv@vtr.net 